\title{
Measurements of WW, ZZ cross section and search for anomalous quartic gauge couplings at D0
}

\author{
Sudeshna Banerjee ${ }^{* \dagger}$ \\ Tata Institute of Fundamental Research \\ E-mail: sudeshna.banerjee@cern.ch \\ D0 Collaboration, Fermi National Laboratory
}

\begin{abstract}
We present measurements of anomalous quartic gauge couplings and $Z Z$ production cross section in $p \bar{p}$ collisions at $1.96 \mathrm{TeV}$ with Run II data ranging from $8.6 \mathrm{fb}^{-1}$ to $9.9 \mathrm{fb}^{-1}$ collected by the D0 detector at the Fermilab Tevatron Collider. A search for anomalous quartic gauge couplings (AQGC) involving photons and $\mathrm{W}$ bosons is performed using a sample of di-electron events with a large amount of missing energy. No excess above the standard model (SM) background has been observed and limits on the anomalous parameters $a_{0}^{W}$ and $a_{C}^{W}$ are extracted. In the $Z Z$ cross section measurement, we examine the final states eеeе, ее $\mu \mu$, and $\mu \mu \mu \mu$. Based on selected data the measured cross section for $Z Z$ is consistent with the SM expectation. We extend this result to search for the SM Higgs boson between 115 and $200 \mathrm{GeV}$.
\end{abstract}

The European Physical Society Conference on High Energy Physics -EPS-HEP2013

18-24 July 2013

Stockholm, Sweden

* Speaker.

${ }^{\dagger}$ A footnote may follow. 
$W W$ and $Z Z$ production are interesting and important because their production cross sections are an order of magnitude smaller than those of the $W$ and the $Z$ bosons. Also, the Higgs boson decays to $W W$ and $Z Z$ events with substantial branching ratios. Therefore, SM production of $W W$ and $Z Z$ events are backgrounds for the Higgs events. If the measured values of the cross sections show deviations from the SM values then they will signify evidence of new physics. The D0 detector which has been used for both searches is describd in [1].

\section{Search for AQGCs}

Using $W W$ events one can probe trilinear $(V V V)$ and quartic $(V V V V)$ gauge couplings $(V=$ $W, Z, \gamma)$ which could show what goes on during the process of electroweak symmetry breaking and also can be a signature of new physics. Figures 1 (a) and (b) show processes involving trilinear and quartic gauge couplings. In the quantum electrodynamics process $p \bar{p} \rightarrow W W p \bar{p}$, the $W$ pair is produced via photon exchange which radiate directly from $p$ and $\bar{p}$. The SM cross section for this process is $\sim 3 \mathrm{fb}$. An enhancement in the value of the cross section by 10 to 100 times can occur due to various beyond SM processes, e.g. extra dimensions [2].

Results are presented here on AQGCs assuming that triple gauge couplings $W W \gamma$ are at their SM values. Deviations from the SM values of these copulings have been constrained by measurements done earlier by D0 and others [3]. Parametrization of AQGCs is based on [4] and only the lowest dimension operators that have the correct Lorentz invariant structure and fulfill the $\mathrm{SU}(2)_{C}$ custodial symmetry are considered. Such operators involving two $W$ bosons and two photons are of dimension six:

$$
L_{6}^{0}=\frac{-e^{2}}{8} \frac{a_{0}^{W}}{\Lambda^{2}} F_{\mu \nu} F^{\mu v} W^{+\alpha} W_{\alpha}^{-}, L_{6}^{C}=\frac{-e^{2}}{16} \frac{a_{C}^{W}}{\Lambda^{2}} F_{\mu \alpha} F^{\mu \beta}\left(W^{+\alpha} W_{\beta}^{-}+W^{-\alpha} W_{\beta}^{+}\right)
$$

Here $F_{\mu \nu}$ is the electromagnatic field strength tensor and $W_{\alpha}^{ \pm}$are the $W^{ \pm}$boson fields. The anomalous parameters, $a_{0}^{W}$ and $a_{C}^{W}$ are 0 in SM. To prevent a violation of unitarity at high energies, a form factor, $a_{i}^{W} \rightarrow \frac{a_{i}^{W}}{\left(1+M_{\gamma \gamma}^{2} / \Lambda_{\text {cutoff }}^{2}\right)^{2}}$ is used where $M_{\gamma \gamma}$ is the invariant mass of the two photons and $\Lambda_{\text {cutoff }}$ is the scale of new physics. Two typical values of this scale chosen for this analysis are 0.5 and 1.0 $\mathrm{TeV}$.

In this search the signal events are $p \bar{p} \rightarrow W W p \bar{p}$. The bacground events can be classified as diffractive and non-diffractive. The diffractive events are due to $W W$ and $l l$ production through photon exchange or double pomeron exchange. Signal and diffractive background events are modeled using Forward Physics Monte Carlo [5]. Their passage through the D0 detector is modeled using a GEANT3 based detector simulation package followed by the D0 reconstruction package. Signal events are generated by scanning one of $a_{0}^{W}$ or $a_{C}^{W}$ with or without a form factor. Nondiffractive backgrounds can be classified into two types, physics backgrounds containing $Z / \gamma+$ jets, $t \bar{t}$, diboson $(W W, W Z, Z Z)$, and instrumental backgrounds containing $W+$ jets and multijets. These were modeled using PYTHIA or ALPGEN + PYTHIA [6]. The amount of multijet background is fully determined from data.

The production cross section of a pair of $\mathrm{W}$ bosons, $p \bar{p} \rightarrow W W$ was measured by $\mathrm{D} 0$ in the analysis of $H \rightarrow W W \rightarrow l v l v$ decays [7]. Since the search for AQGC involves the same final state $l v l v$, a similar search strategy is followed, but the event selection criteria are tighter. Results are 


\begin{tabular}{|l|r|r|}
\hline & Preselection & Final selection \\
\hline Data & 572700 & 946 \\
Total Background & $576576 \pm 11532$ & $983 \pm 108$ \\
Signal & 12.2 & 11.6 \\
\hline
\end{tabular}

Table 1: Signal $\left(a_{0}^{W} / \Lambda^{2}=5 \times 10^{-4} \mathrm{GeV}^{-2}\right.$, without form factor), background and data yields for AQGC events.

presented here on AQGC signal search in the $W W \rightarrow e v e v$ decay channel only. In the $p \bar{p} \rightarrow W W p \bar{p}$ decays the final state proton and antiproton remain intact but are not detected since they travel in the very forward or backward direction. The two electrons from $W$ decays are central and are boosted. Therefore, events are selected with two opposite sign electrons, and no jet with $p_{T}>20$ $\mathrm{GeV}$ and $|\eta|<2.4$. The pseudorapidity $\eta$ is defined as $\eta=-\ln (\tan (\theta / 2))$, where $\theta$ is the polar angle relative to the proton beam direction. The first and the second electron are required to have $p_{T}$ greater than $15 \mathrm{GeV}$ and $10 \mathrm{GeV}$ respectively and their invariant mass is required to be greater than $15 \mathrm{GeV}$. At least one of the electrons should be in the central electromagnetic calorimeter. To correct for mismodeling of the electron reconstruction and trigger efficiencies, scale factors are applied to the $p_{T}$ distribution of $Z$ bosons in Monte Carlo samples at the preselection stage to match the data. Dependence of the scale factors on the numbers of reconstructed jets in the event is taken into account.

The $p_{T}$ distribution of $W$ bosons is weighted to match the measured $Z$ boson $p_{T}$ spectrum, corrected for the differences between the $W$ and $Z p_{T}$ spectra predicted in NNLO QCD. Figure 2 (a) shows the $p_{T}$ distribution of the leading electron after preselection. Boosted decision trees (BDT) are used to eliminate the contribution from $Z / \gamma^{*}+$ jets background that is dominant after preselection. The input variables to the BDT are kinematic variables related to the two electrons and their opening angle. The most discriminating variable is the transverse mass of the $\mathbb{H}_{T}$ and the dielectron pair, $M_{T}\left(e e, Z_{T}\right)=\sqrt{\left(2 \cdot p_{T}^{e e} \cdot Z_{T} \cdot\left[1-\cos \Delta \phi\left(e e, Z_{T}\right)\right]\right)}$. The cut on the BDT is chosen in such a way that the contributions from $Z / \gamma^{*}+$ jets, $W+$ jets and dibosons are comparable. The $M_{T}$ distribution after the final selection is shown in Fig. 2 (b). The expected and observed numbers of events after the preselection and the final selection are given in Table 1. A final BDT is trained to separate the AQGC signal from all other backgrounds. Additional variables related to electron reconstruction are used as inputs in this case to discard the instrumental backgrounds. The final BDT distribution is shown in Fig. 2 (c). Events with a BDT value above 0.85 are chosen as signal events. Events from all signal points are merged for the BDT training as they have very similar kinematic features.

In this analysis the systematic uncertainties can be divided into two classes. Flat uncertainties are those which affect only the normalization of signal and/or background (e.g. the error on the theory cross section value). Shape uncertainties are those which affect the shape as well as normalization for signal and/or background (e.g. uncertainty in the jet energy scale). Uncertainty due to the processes of Pomeron exchange and DPE normalization is taken as $100 \%$. The other major uncertainties are in multijet normalization and signal cross section, which are of the order of $30 \%$ and $20 \%$ respectively. Since no excess of events above the expected background is seen in the 
$W^{+} W^{-} \rightarrow e^{+} v e^{-} \bar{v}$ decay channel, the data are assumed to be consistent with a background-only model and limits are set using the modified frequentist, $\mathrm{CL}_{s}$, method on the anomalous parameters, $a_{0}^{W}$ and $a_{C}^{W}$ [9]. The test statistic is a log likelihood ratio for background only and signal+background hypothesis. The 95\% C.L. allowed ranges for the anomalous parameter $a_{0}^{W}\left(a_{C}^{W}\right)$ are given in Table 2 assuming $a_{C}^{W}=0\left(a_{0}^{W}=0\right)$. The limits are quoted for the cases without a form factor and with a form factor with $\Lambda_{\text {cutoff }}=1$ or $0.5 \mathrm{TeV}$. The two parameter limits are shown in Fig. 3 for different assumptions about the form factor mentioned above.

\section{ZZ Production Cross section}

The leptonic decays of the $Z$ are used to measure the cross section of $Z Z$ production, i.e. $Z \rightarrow e e$ or $Z \rightarrow \mu \mu$. The dominant tree level diagrams for $p \bar{p} \rightarrow Z / \gamma^{*} Z / \gamma^{*} \rightarrow l^{+} l^{-} l^{+} l^{-}$are shown in Fig. 1 (c), (d). The singly resonant $Z$ boson diagram contributes at low mass. This contribution is expected to be negligible in our analysis. The analysis presented here is based on the full D0 data set of $\sim 9.8 \mathrm{fb}^{-1}$. Signal events are selected by requiring the invariant mass of two highest $p_{T}$ leptons to be greater than $30 \mathrm{GeV}$ for both Z's. If the event contains 4 electrons, at least two of them should be in the central calorimeter. If the event has 4 muons, at least two of the muons should have hits in the muon chambers. If the event has two electrons and two muons then it is required that $\mathrm{dR}(e \mu)>0.2$ for all $e-\mu$ pairs. The $Z Z$ signal acceptance is estimated with PYTHIA [6]. There are three main sources of background, namely, vector boson events with additional photon or jets misreconstructed as leptons, which are mostly $Z+2$ jet events, $Z / \gamma^{*} Z / \gamma^{*}$ events where at least one $Z / \gamma^{*}$ has a mass less than $30 \mathrm{GeV}$ but is reconstructed with a mass above $30 \mathrm{GeV}$, and $t \bar{t}$ events where the leptons in the b-jets pass the isolation cut. The first source of background is estimated from data and the second and third sources are estimated by simulation. The largest contribution to the systematic uncertainty, which is of the order of $10 \%-50 \%$, comes from the instrumental background due to the variation in the jet-lepton misidentification rate. The other major contribution is due to uncertainty in $Z Z$ cross section measurement, which is of the order $\sim 8 \%$. The analysis is divided into 8 subchannels, four in $4 e$, three in $2 e 2 \mu$ and $4 \mu$ decay modes. These are based on the number of electrons in the central, endcap, and inter-cryostat regions. Figure 4 shows a few kinematic features of the $Z Z$ events. $p_{T}$ distributions of the highest, and second highest $p_{T}$ lepton are shown in Fig. 4 (a) and (b) respectively and the $p_{T}$ distribution of the 4-lepton system is shown in Fig. 4 (c).

A total of 13 data events are observed, 5 in the $4 e$ channel, 5 in the $2 e 2 \mu$ channel and 3 in the $4 \mu$ channel. Total number of expected events is $16.8 \pm 1.9$ with contributions of 15.3 \pm 1.9 from the t-channel $Z / \gamma^{*} Z / \gamma^{*}$ production, and $1.5_{+0.2}^{-0.3}$ from the non- $Z Z$ background. The cross section for the $Z / \gamma^{*} Z / \gamma^{*} \rightarrow 4 l$ process is obtained by minimizing the negative log-lilelihood, $-\ln (L)=\sum_{i} \sigma \times B R_{i} \times \alpha_{i} \times \varepsilon_{i} \times \int L . d t+N_{i}^{b k g}-N_{i} \ln \left(\sigma \times B R_{i} \times \alpha_{i} \times \varepsilon_{i} \times \int L . d t\right)$. The effect of systematic uncertainties is added by varying the acceptance and backgrounds by $\pm 1 \sigma$ and adding in quadrature. The cross section of the $p \bar{p} \rightarrow Z / \gamma^{*} Z / \gamma^{*}$ process is found in this analysis to be $1.26_{-0.36}^{+0.44}(\text { stat. })_{-0.15}^{+0.17}$ (syst. $) \pm 0.08$ (lumi). The value of the cross section presented by the NLO theory is $1.7 \pm 0.1 \mathrm{pb}$. The $\gamma^{*}$ contribution can be taken out from the measured $Z / \gamma^{*} Z / \gamma^{*}$ cross section by using the MCFM scale factor. The remaining part is the pure $Z Z$ cross section, which is given by $1.05_{-0.30}^{+0.37}(\text { stat. })_{-0.12}^{+0.14}$ (syst. $) \pm 0.06$ (lumi). The value of the $Z / \gamma^{*} Z / \gamma^{*} \rightarrow 4 l$ cross section 


\begin{tabular}{|l|c|c|}
\hline Cutoff & Expected allowed range $\left[\mathrm{GeV}^{-2}\right]$ & Observed allowed range $\left[\mathrm{GeV}^{-2}\right]$ \\
\hline$a_{0}^{W}=2, a_{C}^{W}=0$ & & \\
\hline No form-factor & {$[-0.00047,0.00043]$} & {$[-0.00046,0.00043]$} \\
$\Lambda_{\text {cutoff }}=0.5 \mathrm{TeV}$ & {$[-0.0024,0.0025]$} & {$[-0.0024,0.0025]$} \\
$\Lambda_{\text {cutoff }}=1.0 \mathrm{TeV}$ & {$[-0.00096,0.00092]$} & {$[-0.00097,0.00089]$} \\
\hline$a_{0}^{W}=0, a_{C}^{W}=2$ & & \\
\hline No form-factor & {$[-0.0016,0.0016]$} & {$[-0.0016,0.0015]$} \\
$\Lambda_{\text {cutoff }}=0.5 \mathrm{TeV}$ & {$[-0.0092,0.0090]$} & {$[-0.0094,0.0092]$} \\
$\Lambda_{\text {cutoff }}=1.0 \mathrm{TeV}$ & {$[-0.0035,0.0033]$} & {$[-0.0035,0.0033]$} \\
\hline
\end{tabular}

Table 2: Expected and observed 95\% CL allowed ranges on $a_{C}^{W}=2$, assuming $a_{0}^{W}$ is zero and vice versa for different assumptions on the form-factor.
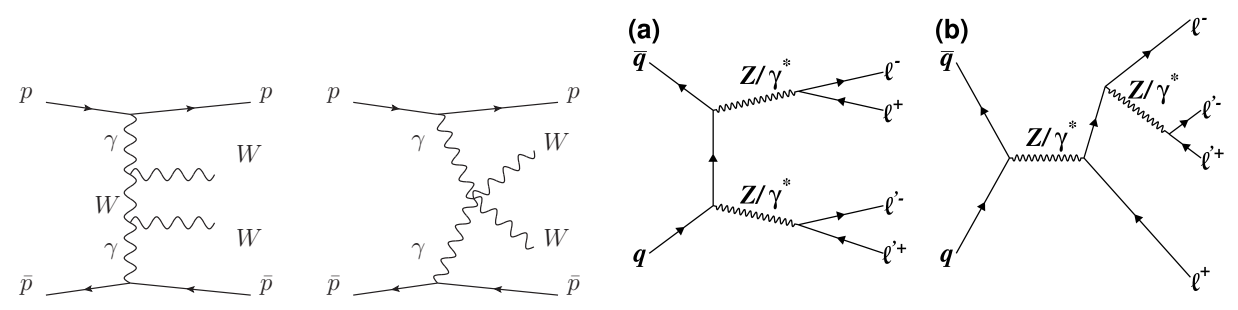

Figure 1: $W$ boson pair production via photon exchange with a) triple, $W W \gamma$ and b) quartic, $W W \gamma \gamma$ couplings. Feynman diagrams for $Z Z$ production through c) t-channel tree level process and d) the singly resonant process.

can be combined with the cross section of the $Z / \gamma^{*} Z / \gamma^{*} \rightarrow l l v v$ channel to give the total $Z / \gamma^{*} Z / \gamma^{*}$ leptonic cross section as $1.32_{-0.25}^{+0.29}$ (stat.) \pm-0.12 (syst.) \pm 0.04 (lumi). This value is in agreement with the SM value of $1.4 \pm 0.1 \mathrm{pb}$ (NLO). Figure 4 (d) shows the various measurements of the $p \bar{p} \rightarrow Z Z$ cross section including the measurement described in this report.

\section{Higgs search}

A new particle, consistent with a SM Higgs boson has been discovered by the ATLAS and CMS experiments at $125 \mathrm{GeV}$ in the $H \rightarrow Z Z$ and $H \rightarrow \gamma \gamma$ decay modes [8]. The D0 ZZ cross section measurement can be an alternate method to search for the Higgs boson. For events with small $E_{T}(<30 \mathrm{GeV})$ the 4-lepton invariant mass can be used as the main discriminant between signal and background. For events with large $\not_{T}(>30 \mathrm{GeV}), \not_{T}$ itself can be used as a discriminant. Figure 5 (a) and (b) show the distributions of the 4-lepton invariant mass and the $\not_{T}$ respectively, from 9.8 $\mathrm{fb}^{-1}$ of D0 data. At $125 \mathrm{GeV} 0.14$ Higgs boson events are expected. Since the observed number of events are cosistent with the background, a limit on the Higgs mass is calculated using the modified frequentist approach where log likelihood ratio (LLR) is taken as the test statistic [9]. Figure 5 (c) shows the $95 \%$ exclusion contour for the ratio $\sigma_{h} / \sigma_{S M}$ versus the Higgs masses with the condition $\frac{C L_{s+b}}{C L_{b}}=C L_{s} \leq 0.0$ along with the associated LLR distribution (Fig. 5 (d)). At Higgs masses of 115, 

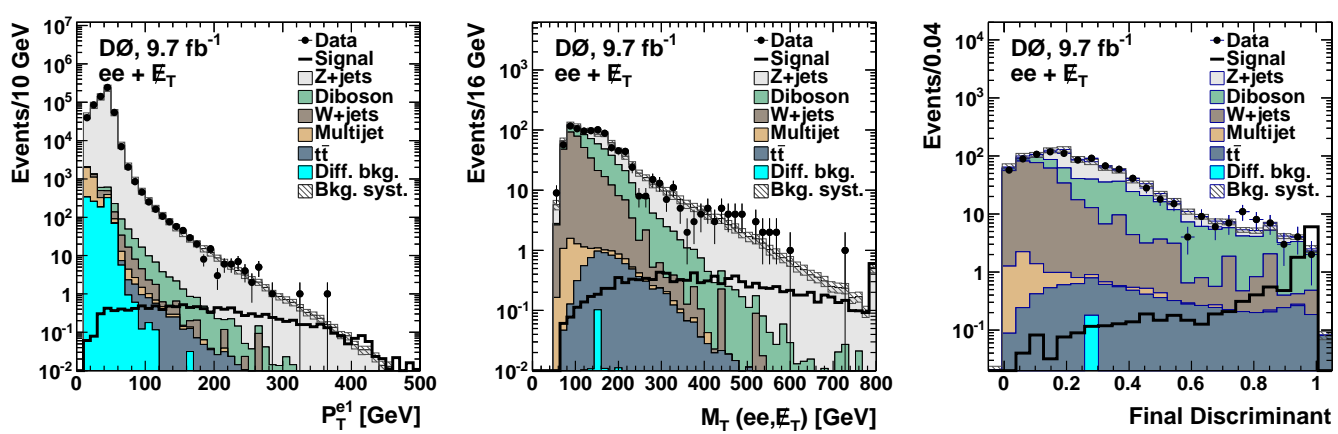

Figure 2: (colour online) The (a) leading electron $p_{T}$ at preselection, (b) transverse mass of the $Z_{T}$ and the two electrons after final selection, (c) BDT distribution after final selection. The hatched bands show the total systematic uncertainty on the background prediction. For the signal distribution $a_{0}^{W} / \Lambda^{2}=5 \times 10^{-4}$ $\mathrm{GeV}^{-2}$ and no form factor is assumed.
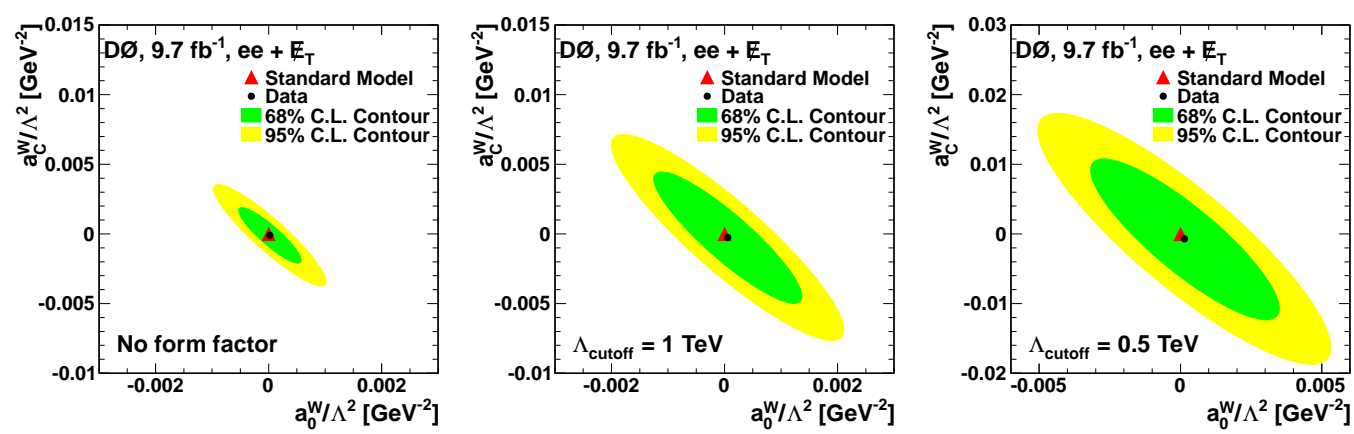

Figure 3: (color online) Two-parameter 68\% and 95\% C.L. limits with different assumptions about the signal, (a) no form factor, or a form factor with (b) $\Lambda_{\text {cutoff }}=1$ or (c) $0.5 \mathrm{TeV}$.

120 , and $125 \mathrm{GeV}$ the observed limits are $57.3 \times, 54.9 \times$, and $42.3 \times \mathrm{SM}$ respectively and the expected limits are $78.9 \times, 60.6 \times$, and $42.8 \times$ SM respectively.

\section{Summary}

D0 has placed the limits $\left|\frac{a_{0}^{W}}{\Lambda^{2}}\right|<0.0025 \mathrm{GeV}^{-2}$ with $\left|\frac{a_{C}^{W}}{\Lambda^{2}}\right|=0$ and $\left|\frac{a_{C}^{W}}{\Lambda^{2}}\right|<0.0092 \mathrm{GeV}^{-2}$ with $\left|\frac{a_{0}^{W}}{\Lambda^{2}}\right|=0$ on the anomalous $W W \gamma \gamma$ quartic gauge boson couplings with $\sim 9.8 \mathrm{fb}^{-1}$ of data. Here it is assumed that $\Lambda_{\text {cutoff }}=0.5 \mathrm{TeV}$ [10]. D0 has also improved the measurement of the cross section of the process $p \bar{p} \rightarrow Z / \gamma^{*} Z \gamma^{*}$ to be $1.26_{-0.36}^{+0.44}$ (stat.) ${ }_{-0.15}^{+0.17}$ (syst.) \pm 0.08 (lumi) [11].

\section{References}

[1] V. M. Abazov et al. (D0 Collaboration), Nucl. Instrum. Methods Phys. Res., Sect. A 565, 463 (2006); M. Abolins et al., R. Angstadt et al., Nucl. Instrum. Methods Phys. Res., Sect. A 622, 298 (2010).

[2] E. Chapon, C. Royon, and O. Kepka, Phys. Rev. D 81, 074003 (2010), P. J. Dervan, A. Signer, W. J. Stirling, and A. Werthenbach, J. Phys. G 26, 607 (2000), O. J. P. Eboli, M. C. Gonzalez-Garcia, S. M. Lietti, and S. F. Novaes, Phys. Rev. D 63, 075008 (2001). 

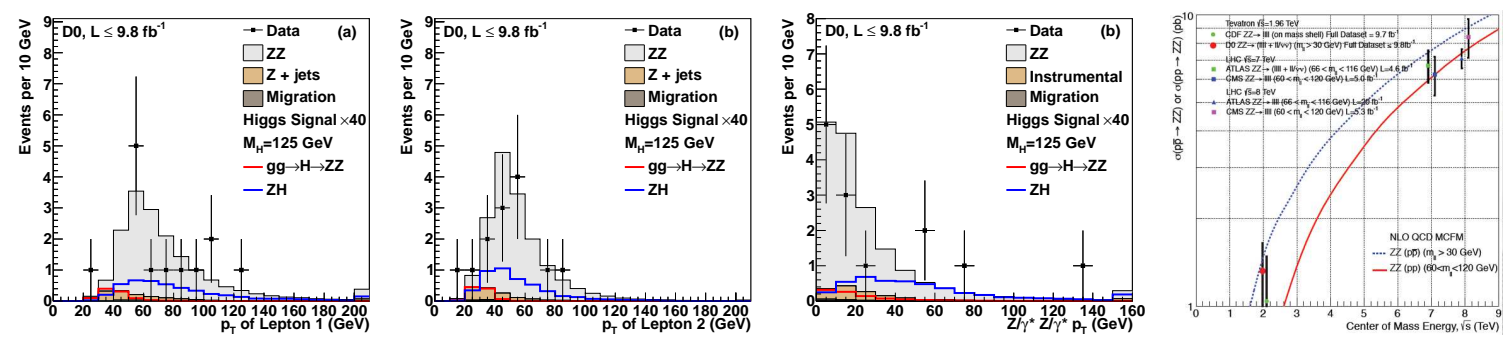

Figure 4: (color online) Distributions of the transverse momentum in data, expected signal, and backgrounds for the (a) highest-pT , (b) secondhighest- pT leptons and (c) the four-lepton system in each event. The Higgs boson signal for $M_{H}$ of $125 \mathrm{GeV}$ is shown scaled by a factor of 40. (d) Current and earlier measurements of the $p \bar{p} \rightarrow Z Z$ cross section.
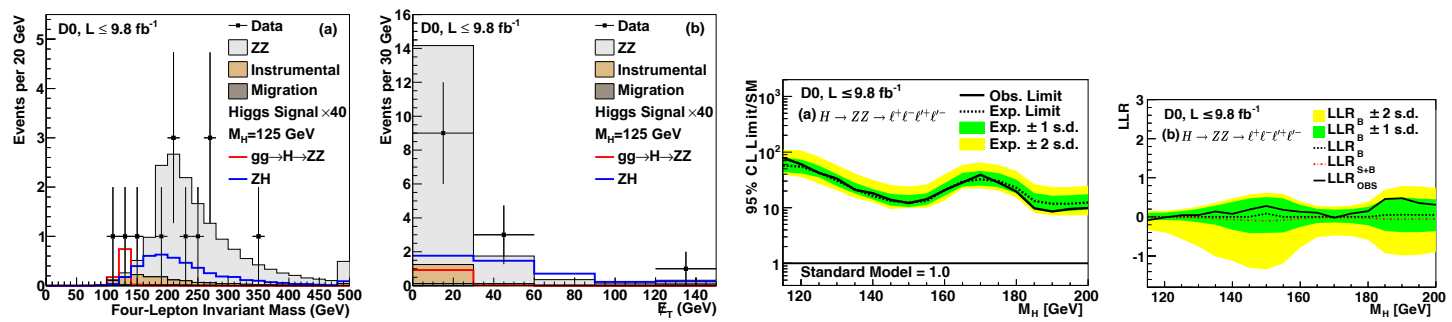

Figure 5: (color online) Distributions of (a) the four lepton invariant mass and (b) the $\not_{T}$ in data, and of expected signal and background. The Higgs boson signal for $M_{H}$ of $125 \mathrm{GeV}$ is shown scaled by a factor of 40. The (c) expected and observed 95\% C.L. upper limits on the SM Higgs boson production cross section relative to the value expected in the SM, and the (d) log-likelihood ratio for all four lepton channels combined.

[3] V. M. Abazov et al. (D0 Collaboration), Phys. Lett. B 718, 451 (2012), G. Aad et al. (ATLAS Collaboration), Phys. Rev. D 87, 112001 (2013); 87, 112003 (2013), S. Chatrchyan et al. (CMS Collaboration), Eur. Phys. J. C 73, 2283 (2013).

[4] G. Belanger and F. Boudjema, Phys. Lett. B 288, 210 (1992). In the present study, the $a_{0}^{Z}$ and $a_{0}^{Z}$ parameters are assumed to be zero, R. A. Diaz and R. Martinez, Rev. Mex. Fis. 47, 489 (2001).

[5] M. Boonekamp et al., arXiv:1102.2531v1, http://cern.ch/fpmc.

[6] T. Sjostrand, S. Mrenna, and P. Skands, J. High Energy Phys. 05 (2006) 026; we use versions 6.319 and 6.413, M. L. Mangano, F. Piccinini, A. D. Polosa, M. Moretti, and R. Pittau, J. High Energy Phys. 07 (2003) 001; we use version 2.11.

[7] V. M. Abazov et al. (D0 Collaboration), Phys.Rev. D 88 (2013) 052006.

[8] G. Aad et al. (ATLAS Collaboration), Phys. Lett. B 716, 1 (2012), S. Chatrchyan et al. (CMS Collaboration), Phys. Lett. B 716, 30 (2012).

[9] T. Junk, Nucl. Instrum. Methods Phys. Res. A 434, 435(1999), A. Read, J. Phys. G 28, 2693 (2002), W. Fisher, FERMILAB-TM-2386-E (2006).

[10] Phys. Rev. D 88, 012005 (2013).

[11] Phys.Rev. D 88 (2013) 032008. 